\title{
Finite size effects in cooperative molecular motors
}

\author{
Sencer Taneri ${ }^{\mathrm{a}, *}$, M. Cemal Yalabik ${ }^{\mathrm{b}}$ \\ ${ }^{a}$ Feza Gürsey Institute P.O.Box 6, 81220 Çengelköy, Istanbul, Turkey \\ ${ }^{\mathrm{b}}$ Department of Physics, Bilkent University, 06533 Bilkent, Ankara, Turkey
}

Received 12 March 2003

\begin{abstract}
We have studied the ballistic and damped dynamics of finite size cooperative molecular motors. We study the effect of finite size on average quantities such as sliding velocity, as well as the statistics of fluctuations in these quantities. We observed stalling for the over damped case.

(c) 2003 Elsevier B.V. All rights reserved.
\end{abstract}

PACS: 05.20. $-y ; 87.15 .-v$

Keywords: Motor; Protein; Cooperative

\section{Introduction}

The study of motor proteins is significant because the biological collective action of such motors (as in the case of muscle contraction) generates very interesting phenomena which has also generated theoretical interest [1-3]. Cooperative molecular motors consist of multi-motor filaments which slide on micro-tubules. In most theoretical studies of systems of such motor proteins, the size of the system is assumed to be very large, or infinite, so that the collection may be considered as a rigid backbone sliding with a constant velocity on a ratchet like potential. (We will call these individual motor proteins as "particles" for consistency with previous literature.) Such large size, in the presence of a ratchet potential incommensurate with inter-motor spacing, implies a uniform distribution of the particles over the period of the potential, and simplifies the analysis considerably. The uniform distribution also eliminates the fluctuations in the effective force experienced by the chain, resulting in uniform motion.

\footnotetext{
* Corresponding author. Tel.: +90-216-308-9432/36; fax: +90-216-308-9427.

E-mail address: taneri@gursey.gov.tr (S. Taneri).
} 
In this work, we look at a molecular motor system which is made up of a finite number of motors. The force experienced by the motor filament as it moves on the ratchet potential is no longer uniform. This would then indicate a non-uniform motion of the chain, and we have studied the characteristics of this motion for the damped as well as the under damped ballistic case, where the mass of the system would also be expected to play a role. Very long as well as very massive proteins would be expected to yield results close to the studies on infinite length proteins, which act as a check on our results. The two-state model we have used includes a symmetric saw-tooth potential for the track on which the particles are attached, and a constant potential to describe the physical aspects of the detached state of the particles [2]. The state of the particles change as a result of thermal excitations. The detailed balance is broken through the one-way Adenosinetriphosphate (ATP) excitations [1-4]. Therefore, one can see spontaneous motion even when the saw-tooth potential is symmetrical. The problem has been studied by Jülicher and Prost [1,3] for the case where the backbone and ratchet potential are incommensurate and infinite in extent which enables an analytic analysis of the statistics of the infinite system.

In these studies, the relationship between the excitation amplitude and spontaneous velocity of the backbone and also the velocity dependence of the motor force for different excitation amplitudes have been investigated. The motion of the motor when it is made up of finite length "particles" in presence of noise has also been studied [5]. It was reported that there is a complex dependence of the average velocity on both the size and the average distance of the particles. The variations in system behavior in the presence of deformations which lead to enhanced correlation among the motor states has also been studied by the present authors [6]. In this study the basic mechanism of motion was the spontaneous symmetry breaking due to ATP presence in the symmetric ratchet potential.

Short-chain length limits has been studied extensively $[7,8]$. In these studies coupled motors with external driving force, in the absence of ATP but with asymmetric periodic potentials are taken into account. The dynamics is analyzed for different coupling strengths above the critical coupling [8]. Commensurate case is used to study the coupling constant and particle number dependence of velocity in presence of synchronous and asynchronous external driving force [7]. Our study here will be an extension of above studies for infinitely large coupling limit in presence of ATP. In the present work, we study motors made up of a small number of particles, in which case a continuous distribution of particles on the potential period can no longer be assumed. In fact, for such small systems, the sliding filament is expected to feel a set of discrete values of force. We simulate these finite systems through the Langevin equation [9-12] both for the over damped case where the total force of the system is assumed to be proportional to its velocity and also for the "finite mass" case where the inertia of the system cannot be neglected (we will refer to this case as the "ballistic" problem). We have included the mass (therefore the acceleration) terms associated with the equation of motion which is normally neglected in the high damping limit. Our motivation was driven by the fact that relatively large fluctuations in velocity appear with relatively small size motors, and corresponding accelerations may be large enough to alter the dynamical behavior qualitatively. 
We recover the previously reported results in the infinite chain length limit. While the infinite (in mass or size) system implies a perfect statistical averaging, fluctuations appear in finite systems. We display the fluctuations in the sliding velocity as a function of time, and associate a time constant with them.

\section{Model}

A symmetric saw-tooth potential for the track filament and a higher constant potential for the backbone for the two state system [2] is chosen (Fig. 1). The probability for particles to change their potential states from the upper level to the lower one and vice versa is introduced as described below.

The transition rates between the two states for the $i$ th particle at coordinate $x$ are chosen as

$$
\omega_{1}\left(x_{i}\right)=\omega_{2} \exp \left[\left(E_{1}\left(x_{i}\right)-E_{2}\right) / k T\right]+\Omega\left(x_{i}\right),
$$

where $E_{1}$ and $E_{2}$ are as indicated in Fig. 1 and $\omega_{1}\left(x_{i}\right)$ is the transition rate from the symmetric saw-tooth potential state to the detached state. The transition rate for the reverse process $\omega_{2}$ is chosen as 1 . Here, $k$ and $T$ denote the Boltzmann constant and the temperature. The additional transition rate $\Omega\left(x_{i}\right)$ out of the symmetric saw-tooth state is caused by the ATP excitations, and is assumed to be present at certain points on the track, as shown in Fig. 1. We will call the magnitude of this rate $\Omega\left(x_{i}\right)$ as "the excitation amplitude" for consistency with previous work [1]. We take $\Omega$ a value sufficiently large so that all particles passing through the ATP regions are effectively transferred to the detached state.

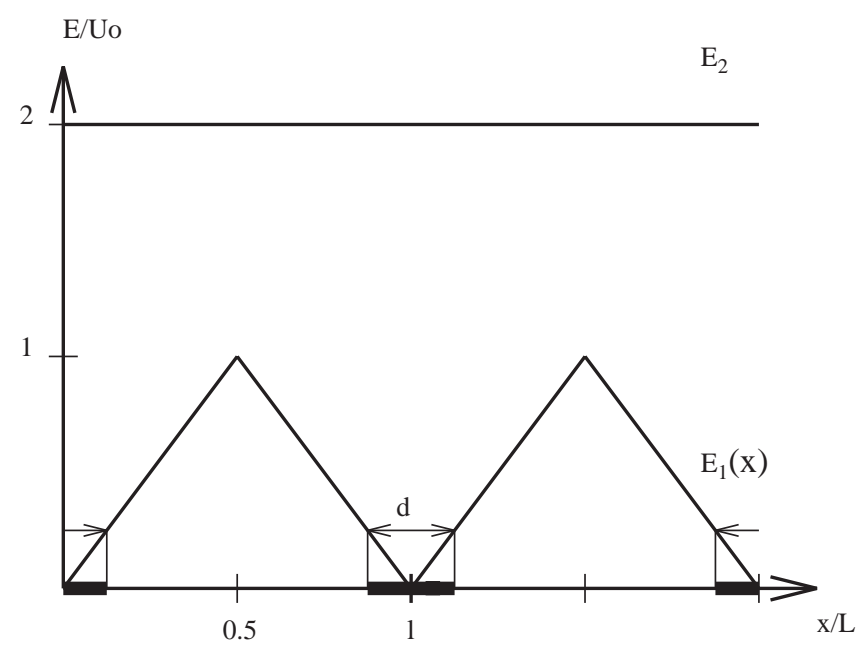

Fig. 1. A simple diagram of the $E 1(x)$ and $E 2$ potentials. The width of the region where ATP excitation occurs is $d=0.1$ and $U_{0}$ is the height of the symmetric saw-tooth potential with respect to which length and the energy are scaled. 
Although we have chosen a symmetrical potential for the saw-tooth potential state, it was shown in Ref. [1] that a spontaneous symmetry breaking leads to sustained motion in one direction. The motivation for the choice of a symmetric potential comes from our aim of studying fluctuations of velocity in the finite system. The fluctuation themselves are then simply between the two different symmetric motions of the system. The motion of the motor is governed by the Langevin equation, with the mass term included.

$$
N M \ddot{x}=-N \gamma \dot{x}-\sum_{i} \frac{\mathrm{d} U\left(x_{i}\right)}{\mathrm{d} x_{i}}-\sum_{i} \xi_{i}(t)
$$

with the assumption that all particles have the same mass and that the inter-particle distance does not change so that the velocity $\dot{x}$ is the same for all particles. Here, $N$ is the number of particles, $x_{i}$ is the position of the $i$ th particle, $U\left(x_{i}\right)$ is either $E_{1}\left(x_{i}\right)$ or $E_{2}$ depending on which potential level the particle is in. Here, $\xi_{i}(t)$ is Gaussian white noise [10] with correlation function $\left\langle\xi_{i}(t) \xi_{j}\left(t^{\prime}\right)\right\rangle=2 D \delta_{i, j} \delta\left(t-t^{\prime}\right)$ with zero mean and $2 D$ is the noise strength with $D=\gamma k T / N M^{2}$ [10]. All of the remaining forces are lumped into the $-N \gamma \dot{x}$ damping term which represents the sum of viscous friction forces and possibly an externally applied force proportional to speed. We limit our study to number of particles $N=1, \ldots, 10$.

We use unitless quantities such that lengths are scaled by the period of the symmetric saw-tooth potential $L$, energies by the magnitude of the potential $U_{0}$ and masses by the mass of a protein particle $M_{0}$. The time variable $\tau$ is then scaled by $\tau_{0}=\sqrt{M_{0} L^{2} / U_{0}}$. Then for $M=M_{0}, u=U / U_{0}$ and Eq. (2) can be rescaled and written as

$$
N \ddot{x}=-N \Gamma \dot{x}-\sum_{i} \frac{\mathrm{d} u\left(x_{i}\right)}{\mathrm{d} x_{i}}-\frac{L}{U_{0}} \sum_{i} \xi_{i}(t),
$$

where $\Gamma=\gamma \tau_{0} / M_{0}$. With this scaling, we have chosen the energy of the detached state as $E_{2} / U_{0}=2$ and $k T / U_{0}=1$ for the filament and 0.01 for the environment in our computations. Inter-particle distance $\Delta x$ is taken to be incommensurate with the symmetric saw-tooth potential period as $\sqrt{2} /\{2,4\}$. We choose $M / M_{0}=1.2$ and $\gamma=0.1$.

\section{Calculation}

The numerical computation was started by assigning the motor chain an initial constant velocity. The force acting on the individual particles was then calculated at each time step. This force due to potentials indicated in Fig. 1 is determined by the slope of the potential of the state the particle is in (the force on a detached particle is trivially zero whereas the force on an attached particle is negative the slope of the model symmetric saw-tooth potential). The motor is moved at the initial constant velocity for a time period of $\tau=2$. At the end of this period, it is assumed that the distribution of particle states is sufficiently close to the steady state. 
The state of the particles is changed at each time step by the following procedure: the total probability of any transition in a time interval $\Delta \tau$ is calculated

$$
P=\Delta \tau \sum_{i=1}^{n} \omega_{s}(i),
$$

where $\omega_{s}(i)$ is the transition rate of the $i$ th particle (with $\omega_{s}(i)$ is either $\omega_{1}(i)$ or $\omega_{2}$ depending on the state of the $i$ th particle). The time step $\Delta \tau$ is chosen such that the total probability $P$ is much less than 1 so that the probability of more than one transition in $\Delta \tau$ is negligibly small. In order to comply with this constraint, the analysis is conducted through time steps of $\Delta \tau=2 \times 10^{-3}$. Whether or not a transition will take place is decided at each time step with probability $P$. The particular state which will go through the transition is then chosen randomly with a probability proportional to its transition rate. The total force acting on the filament is determined from the states of the particles. After the initial $10^{3}$ time steps, the total force acting on the motor at each time step due to the symmetric saw-tooth potential is calculated. Use of this total force together with the damping and the random force enables one to solve Eq. (2) numerically [10]. Using the procedure described above at each time step, one can find the velocity of the motor as a function of time. During this process, we use a total of $7.5 \times 10^{4}-7.5 \times 10^{6}$ time steps, depending on the chain length of the motor for good statistical averaging.

In the infinite length case, one finds stable solutions for velocity $V=\mathrm{d} X / \mathrm{d} \tau$. For finite but large $N$, one has finite fluctuations around a $V_{a v}$ value, with intermittinent "switching" to the symmetrical state. On the other hand, smaller $N$ values result in faster fluctuations between $\pm V$ which rapidly average to zero.

For such finite systems the "motor" then moves either in one direction or the other for some period of time, but then reverses its direction and the process continues. The amount of time the motor moves in one direction before reversing its velocity is then an important parameter which characterizes the transport mechanism. We therefore studied the average time $\tau$ that the motor moves in one direction as a function of several parameters. These quantities are characterized by the number and the mass of particles, the temperature and inter-particle distance. Note that there are two kinds of random processes acting on the motor. One is the transitions of motor particles between the attached and detached states and the second is the additive noise-force in Eq. (3). We have studied the system for zero and finite values of additive noise. One can in principle use two different temperature variables for these two types of random events, one for the thermalization of the motor between the two potential energy states and the other for the process generating the Gaussian noise.

We have looked at the finite size coupled-motor system also in the traditional high damping limit (without the mass term). An interesting point about this case is that the velocity of the system is at all times determined by the excitation states of the motors and the overall position of the system with respect to the sawtooth potential. There is therefore, always a finite probability that the system will stop when "accidentally" there are an equal number of attached motor particles on the two types ramps associated with the potential. Our simulations indicate that such states seem to be unstable, but the motion may now start in either of two directions, resulting in a switching of the 
direction of velocity. This effect can be seen pictorially in the plot of the positions (as a function of time) of a number of independent systems, starting with the same initial conditions, but following different trajectories due to the randomness in the thermalization process.

To simulate the over-damped system, we start with an ensemble of systems (made up of 100 elements) and start all of the systems in the ensemble with an initial condition corresponding to a maximum velocity. This is done by artificially assigning attached or detached states to the motors so that all motors facing an incline which would push the system in one direction are all attached, and all the others are detached The individual systems in the ensemble are then allowed to move under the effect of independent random transitions.

For the over-damped simulations, 100 molecular motor systems of same size are artificially given "optimal" starting conditions, i.e., the states of the individual motors are so chosen that the force on the system is maximum. The trajectories of 100 systems made up of $N=20$ and 100 motors are studied. For the smaller size systems, one can observe more rapid switching of velocity direction, although the slopes of the trajectories indicate that the speed of systems moving one way or the other is about the same. For this smaller size, the probability of the system "getting stuck" with zero velocity is larger, and the trajectories indicate that most systems eventually come to rest. For the larger size systems, the switching of the velocity is less frequent, but again the speeds in both directions do not seem to be very different. This then indicates a more stable motion for larger systems. Finally, the spread in the positions of the above-mentioned ensembles of motor-systems in the form of their standard deviation is studied. The smaller systems eventually seem to "get stuck", so that the spread in their positions saturate rather quickly with time. The larger systems, on the other hand, seem to be moving one way or the other, and the spread continues to increase, more or less linearly, within the time scale of our simulations.

\section{Conclusion}

As was discussed earlier, the finite size of cooperative molecular motors has distinctly different behavior in contrast to the infinite system. Our study indicates that velocity fluctuations are significant even for system sizes of $N=10$. The fluctuations were found to diminish exponentially with system size and mass. Our choice of a symmetric saw-tooth potential results in fluctuations that are symmetric in velocity which makes their analysis easier.

The velocity of the 8 particle system as a function of time is shown in Fig. 2 for the noiseless case. As was stated earlier, systems with smaller sizes result in faster fluctuations in velocity switching between $\pm V_{a v}$ values. The average travel time spent in a particular direction (average switching time) is $\tau=3125$.

One can easily assign an average time constant to these fluctuations. The time scale increases exponentially with system size and $1 / \Gamma^{2}$, which is proportional to the mass of the system due to our scaling. These dependencies are shown in Figs. 3 and 4 and 4, with the corresponding fits $\tau \sim \exp (0.68 N)$ and $\tau \sim \exp \left(0.26 / \Gamma^{2}\right)$. 


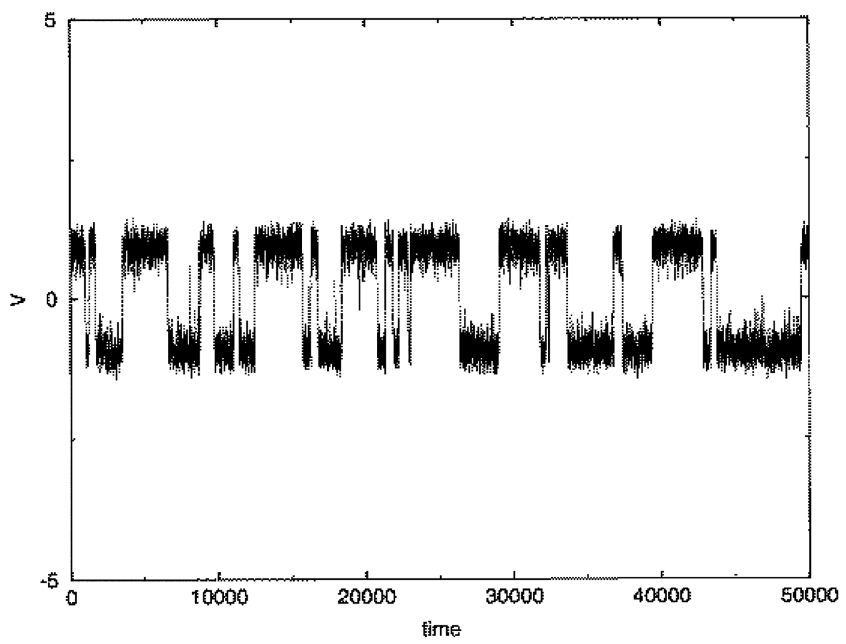

Fig. 2. The time dependence of the velocity for $N=8, \Delta x=\sqrt{2} / 2$ and $\Gamma=0.09$ with $T=0.01$.

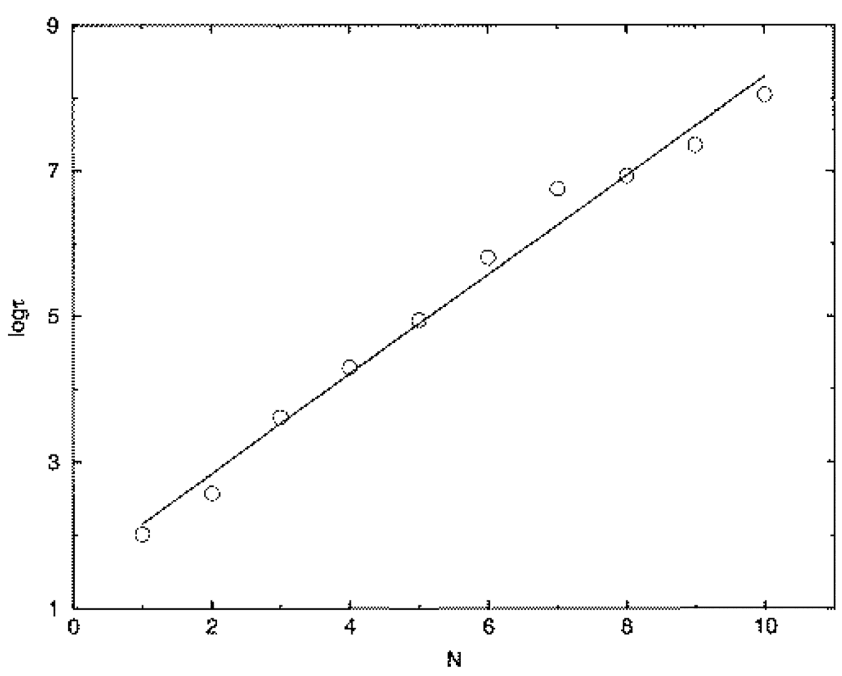

Fig. 3. Logarithm of the switching velocity direction time constant versus system size $\log \tau$ versus $N$ for $\Delta x=\sqrt{2} / 2$ and $\Gamma=0.09$ with $T=0.01$.

The average velocity amplitude versus system size is displayed in Fig. 5 for two different values of inter particle distance. We have chosen irrational values (in relation to the period of the symmetric saw-tooth potential) for the inter particle distance to generate a uniform distribution of coordinates on a period for very long chains in comparison to commensurate case studied by Csahók and his colligues [7]. Apparently even the largest size systems that we considered were not big enough to be completely 


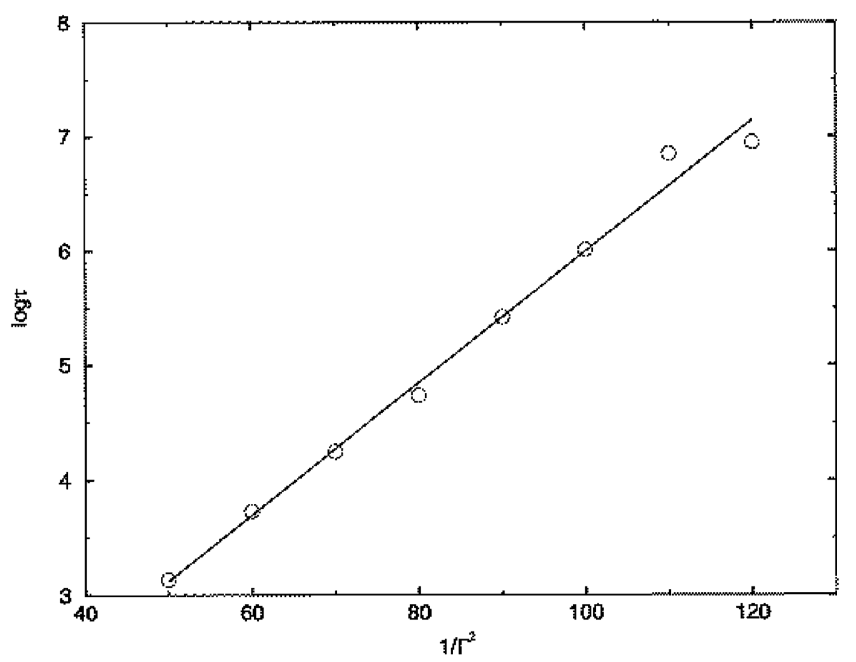

Fig. 4. Logarithm of the switching velocity direction time constant $i$ versus system $\operatorname{size} \log \tau$ versus $1 / \Gamma^{2}$ for $\delta x=\sqrt{2} / 2$ and $N=10$ with $T=0.01$.

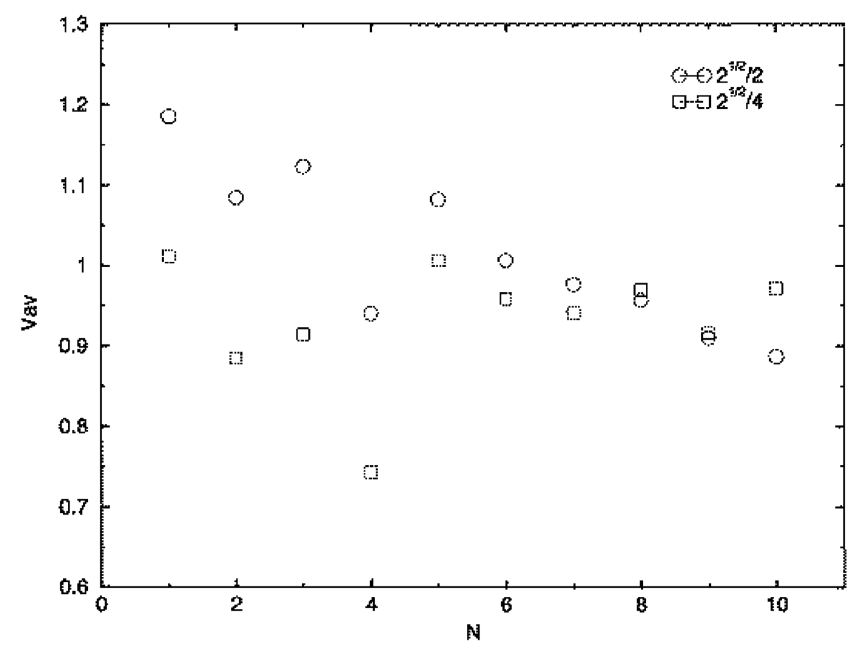

Fig. 5. The speed of the system $V_{a v}$ versus system size $N$ for $\Gamma=0.09$ and $\Delta x=\sqrt{2} / 2$.

independent of particle spacing. However, convergence of larger size systems in Fig. 5 is consistent with the infinite size limit calculated by Jülicher [1]. Variations due to inter particle spacing are largest for the smaller size systems as expected.

The dependence of the average velocity to $\Gamma$ is displayed in Fig. 6. This graph shows the expected decrease of variations with increase of the damping coefficient. Note that the variations due to different $\Delta x$ seemed compressed relative to those in Fig. 5 due to the difference in the vertical scale. 


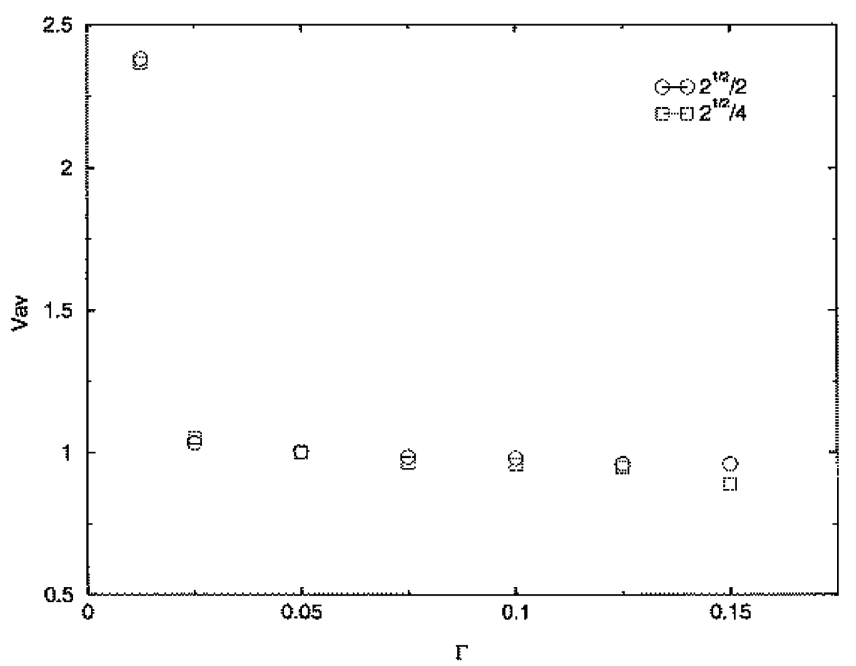

Fig. 6. Speed $V_{a v}$ versus friction coefficient $\Gamma$ for $\Delta x=\sqrt{2} /\{2,4\}$ and $N=10$.

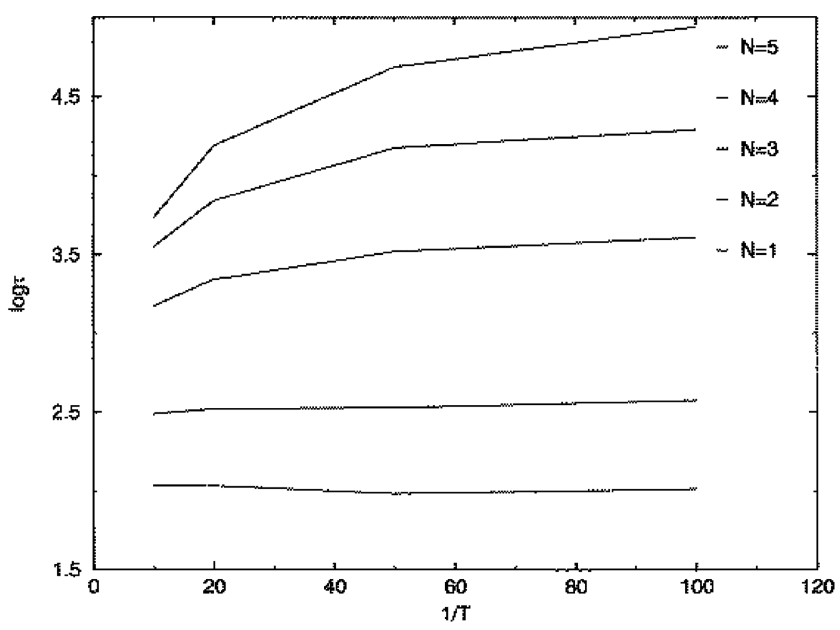

Fig. 7. Switching time $\log \tau$ versus inverse temperature $1 / T$ for $\Delta x=\sqrt{2} / 2$, for system sizes $N=1-5$ and $\Gamma=0.09$.

We have also studied the effect of random force on the system, modeled as a thermal noise. Note that this brings a second (and in our case independent) temperature variable to the analysis in addition to the attachment/detachment process. Figs. 7 and 8 and 8 displays the noise temperature dependence of average switching time. Increase of noise temperature lead to an increase in the thermal excitation rate, thus giving rise to faster fluctuations in velocity. Note that the fluctuations in velocity do not disappear as this noise temperature becomes very small due to the random process associated with 


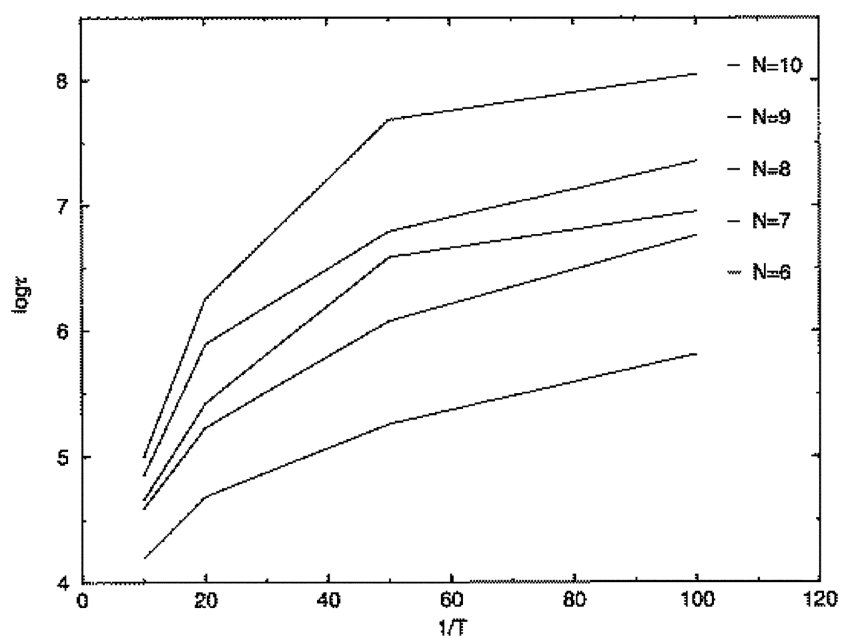

Fig. 8. Switching time $\log \tau$ versus inverse temperature $1 / T$ for $\Delta x=\sqrt{2} / 2$, for system sizes $N=6-10$ and $\Gamma=0.09$.

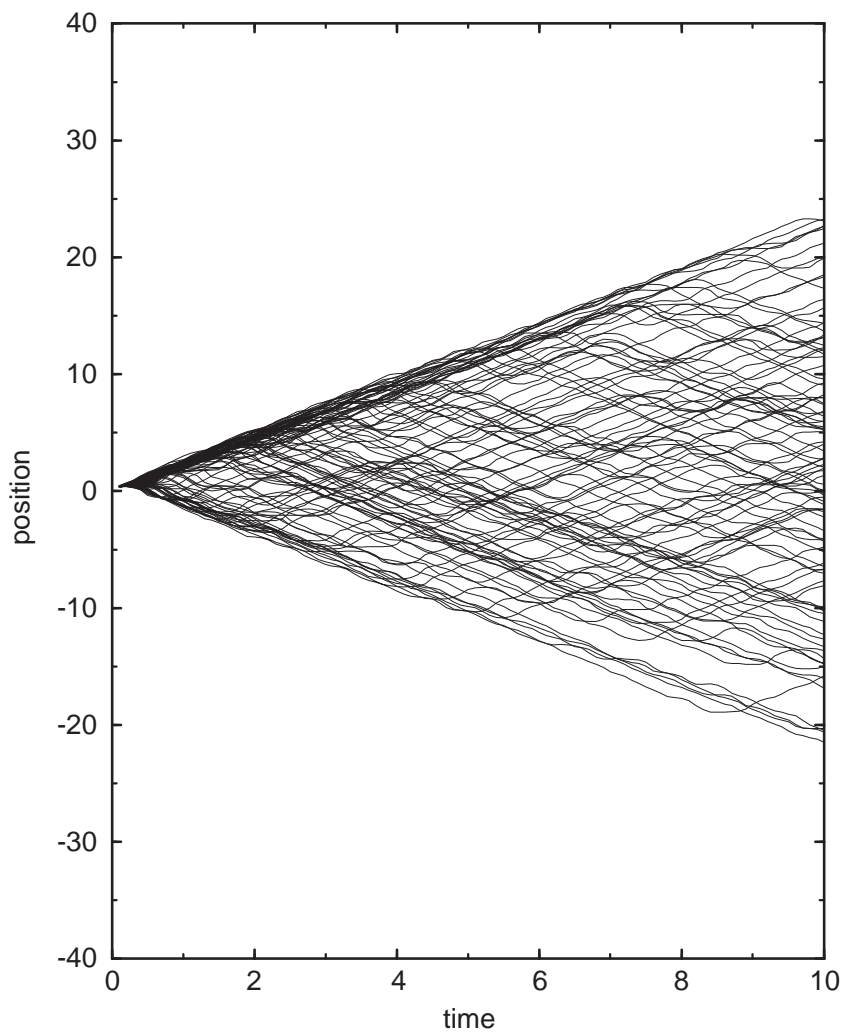

Fig. 9. Position of an ensemble of 100 motor system of size $N=100$ as a function of time. 


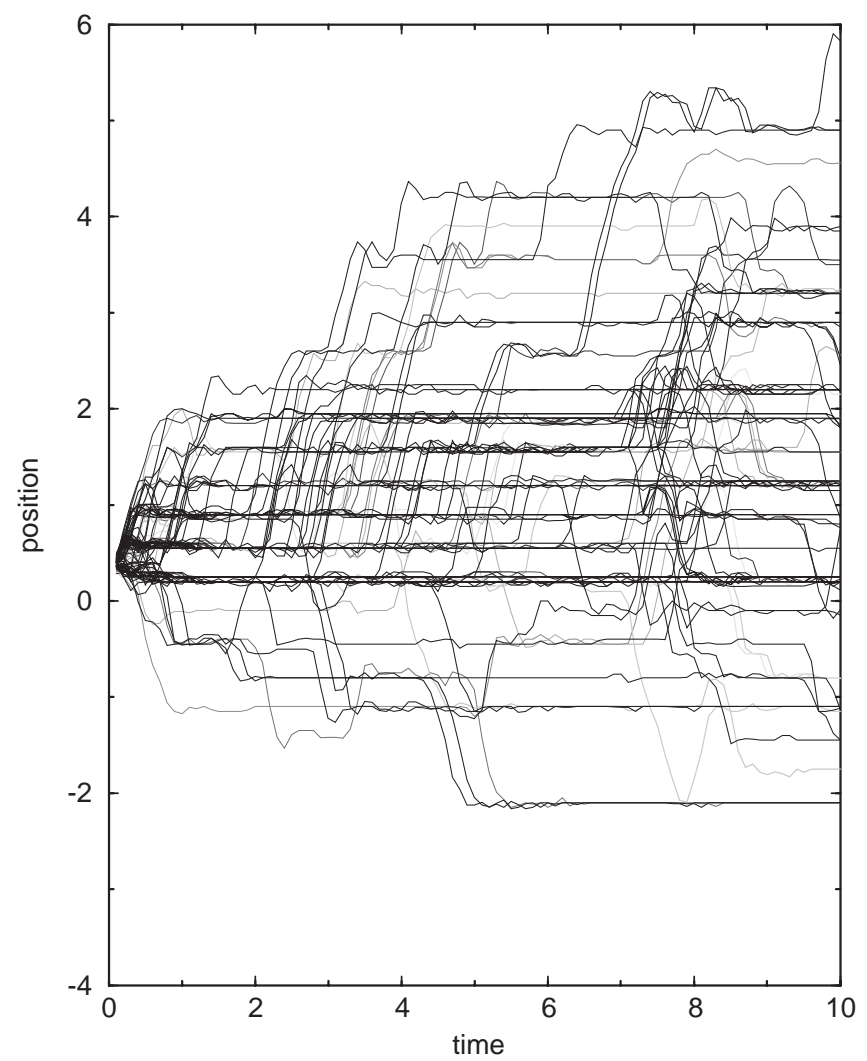

Fig. 10. Position of an ensemble of 100 motor system of size $N=20$ as a function of time.

attachment/detachment. The rate processes associated with the two different random processes do not seem to odd trivially.

For the over damped case, one no longer has any inertial effects but the velocity of the system is proportional to the total force acting on the system. In fact, because of the finite size of the system, the total force acting on the system (and therefore the velocity) can take one of a finite set of discrete values.

There is a finite probability that the total force at a certain time may be zero, and therefore the system may stop. In fact, it may be stated that any such motor will eventually stop due to this finite probability. Such stalling will occur when motor particles will be attached to the ratchet potential, with equal numbers on oppositely inclined ramps. Although it would be expected that such events should have very small probabilities for "reasonably long" chains, our simulations indicate that a system with a size of $N=20$, or even $N=100$ show evidence of such stalling.

Fig. 9 shows the coordinates of the 100 systems of size $N=100$ in the ensemble. A vast majority of the systems move with a constant steady-state speed, but occasionally, a number of systems switch directions, and a few do stop moving. The effect is 


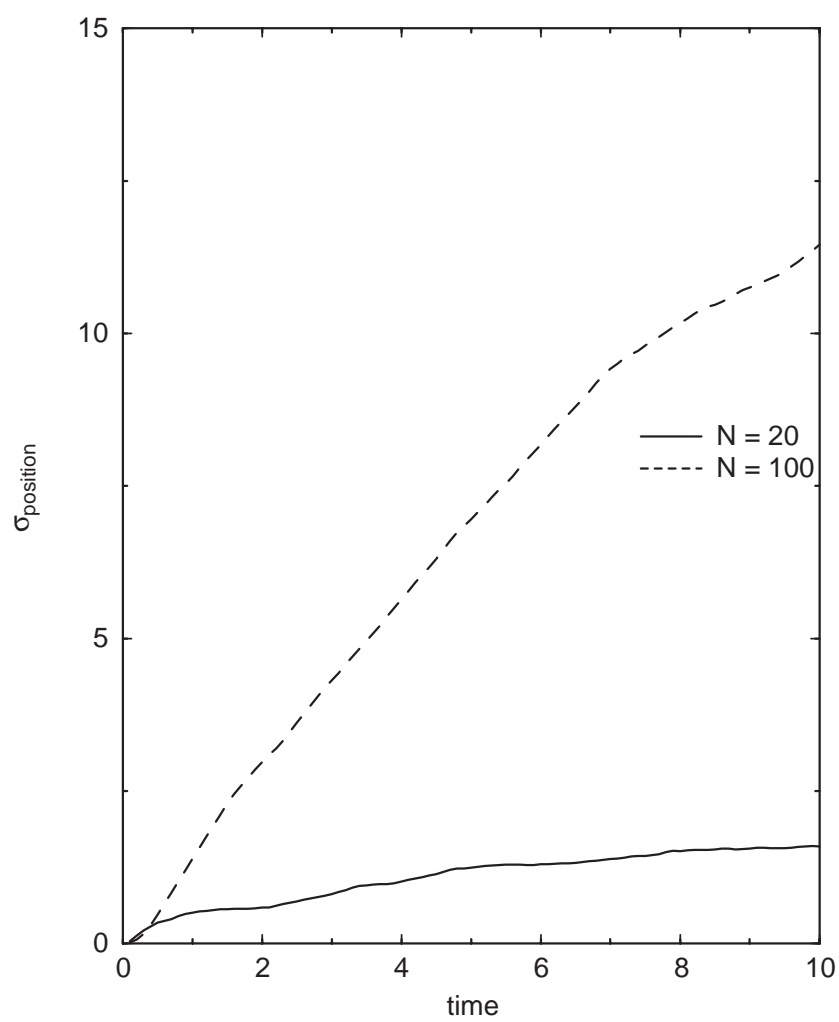

Fig. 11. Deviations of positions of systems in Figs. 9 and 10 versus time.

much more prominent in the ensemble containing systems with sizes $N=20$, as shown in Fig. 10. Almost all of the systems have come to a stop by the end of the simulation period like it did in absence of noise and external driving force in Csahók et al.'s work [7].

Fig. 11 shows the spread in the coordinates of the systems in the two ensembles considered above. The change in the standard deviation of the coordinates of the systems indicate that the $N=20$ systems come to rest rapidly, so that the spread in the coordinated saturates quickly, while for the $N=100$ ensemble such an effect is not apparent in the time scales that were considered.

We have studied the effects of finite size on the dynamics of systems of coupled molecular motors. We have shown that there are profound effects, which result in significant fluctuations in the velocity of the system, and in the over damped case, may result in the complete stalling of the system. The fact that these effects can be seen easily even in system sizes of $N=100$ indicate that results of calculations assuming infinite system sizes may have limitations when applied to actual systems.

In conclusion, we have found out that finite size effects in molecular motor systems manifest themselves in the form of rapid changes in direction of motion. We have 
used a symmetric sawtooth type of track potential, which possibly magnifies this effect. How the details of the shape of the track potential would modify our conclusions is an interesting question.

\section{Acknowledgements}

MCY acknowledges research support from Turkish Academy of Sciences.

\section{References}

[1] F. Jülicher, J. Prost, Phys. Rev. Lett. 75 (1995) 2618.

[2] F. Jülicher, preprint, Physics/9908054, 1999.

[3] F. Jülicher, J. Prost, Phys. Rev. Lett. 78 (1997) 4510.

[4] J. Prost, J.-F. Chauwin, L. Peliti, A. Ajdari, Phys. Rev. Lett. 72 (1994) 2652.

[5] I. Derényi, T. Viscek, Phys. Rev. Lett. 75 (1995) 374.

[6] S. Taneri, M.C. Yalabik, Euro. Phys. J. B 22 (2001) 403-407.

[7] Z. Csahók, F. Family, T. Vicsek, Phys. Rev. E 55 (1997) 5179.

[8] Y. Braiman, F. Family, H.G.E. Hentschel, Phys. Rev. E 53 (1996) R3005.

[9] T.E. Dialynas, K. Lindenberg, G.P. Tsironis, Phys. Rev. E 56 (1997) 3976.

[10] H. Risken, The Fokker-Planck Equation, 1988.

[11] J.H. Li, Z.Q. Huang, Phys. Rev. E 57 (1998) 3917.

[12] R.D. Astumian, M. Bier, Phys. Rev. Lett. 72 (1994) 1766. 